This is the final peer-reviewed accepted manuscript of:

Raisch, M., Genovese, D., Zaccheroni, N., Schmidt, S. B., Focarete, M. L., Sommer, M., Gualandi, C., Adv. Mater. 2018, 30, 1802813.

The final published version is available online at: DOI: $10.1002 / a d m a .201802813$

Rights / License:

The terms and conditions for the reuse of this version of the manuscript are specified in the publishing policy. For all terms of use and more information see the publisher's website.

This item was downloaded from IRIS Università di Bologna (https://cris.unibo.it/)

When citing, please refer to the published version. 


\title{
Highly Sensitive, Anisotropic, and Reversible Stress/Strain- Sensors from Mechanochromic Nanofiber Composites
}

\author{
Maximilian Raisch, Damiano Genovese, Nelsi Zaccheroni, Simon B. Schmidt, \\ Maria Letizia Focarete, Michael Sommer, and Chiara Gualandi*
}

Mechanochromic polymeric systems are intensively investigated for realtime stress detection applications. However, an effective stress-sensing material must respond to low deformation with a detectable color change that should be quickly reversible upon force unloading. In this work, mechanochromic nanofibers made by electrospinning are used to produce mechanochromic nanofiber/poly(dimethylsiloxane) (PDMS) composites with isotropic and anisoptropic response. Due to chain alignment of spiropyran copolymer chains within the nanofibers, only very small strains are required to yield a mechanochromic response. Composites with aligned and isotropic nanofibers show anisotropic and isotropic mechanochromic behavior, respectively. Due to the special substitution pattern of spiropyran in the copolymer, the mechanochromic response of these nanofiber/PDMS composites shows fast reversibility upon force unloading. The outstanding benefit of using highly sensitive mechanochromic nanofibers as filler in composite materials allows the detection of directional stress and strain, and it is a step forward in the development of smart, mechanically responsive materials.

mechanochromic polymers, force responsive species able to give an optical signal, so-called mechanophores, can be dispersed into ${ }^{[6]}$ or covalently bound to a polymer matrix..$^{[7,8]}$ One of the most investigated mechanophore is spiropyran (SP) that, under mechanical stress, isomerizes to the colored merocyanine (MC) form. ${ }^{[9,10]}$ An important challenge that limits applications of SP-based polymers is that the required strain to obtain a response is typically on the order of $100-400 \%$ and hence relatively high, even when the mechanophore is covalently incorporated into a polymer main chain, as underlined by the recent review of Li et al. ${ }^{[11]}$ In addition, macroscopic stress must be able to orient the polymer chains and thus mechanophores in the direction of the applied stress, to finally transduce macroscopic force to the single mechanophore at high strain, and thus induce color change. ${ }^{[12,13]}$ These

Mechanochromic smart materials represent an increasing and interesting category, due to their capability to exhibit mechanically induced color or emission changes, ${ }^{[1,2]}$ and allow usage for imaging mechanical damage and stress/strain distribution. Further possible applications range from tamper proof materials to biological or bio-inspired systems and fundamental studies on polymer adhesion, friction, crack propagation, and plastic flow. ${ }^{[3-5]}$ Changes in the optical properties, as a response to mechanoactivation, are very valuable due to their easy detection and typical high sensitivity. To obtain

M. Raisch, S. B. Schmidt, Prof. M. Sommer

Professur Polymerchemie

Institut für Chemie

Technische Universität Chemnitz

Straße der Nationen 62, 09111 Chemnitz, Germany

Dr. D. Genovese, Prof. N. Zaccheroni, Prof. M. L. Focarete,

Dr. C. Gualandi

Department of Chemistry "G. Ciamician" and INSTM UdR of Bologna

University of Bologna

via Selmi 2, 40126 Bologna, Italy

E-mail: c.gualandi@unibo.it properties can be achieved with semicrystalline thermoplastics and crosslinked or thermoplastic elastomers as matrix materials..$^{[8,14-18]}$ Applications related to glassy and fragile polymers, instead, are limited, ${ }^{6,19,20]}$ since under tension, device failure may occur before mechanophore activation takes place. ${ }^{[21,22]}$ A recent study has shown Förster resonance energy transfer in single conjugated polymer chains in a polystyrene matrix to be suitable to detect very small strains as a change in fluorescence, but $80{ }^{\circ} \mathrm{C}$ was required to render the film ductile. ${ }^{[23]}$

Chain alignment facilitates mechanoactivation at low strain. ${ }^{[13]}$ In the present study, we use an amorphous, alternating spiropyran main chain copolymer that is only moderately ductile in films to produce nanofibers by electrospinning. ${ }^{\text {[24] }}$ Chain orientation within electrospun fibers allows to display a color change after only $5 \%$ strain, which is significantly less compared to mechanochromic bulk samples reported so far. Incorporation of oriented and randomly distributed fibers into a polydimethylsiloxane (PDMS) matrix yield elastomeric, highly sensitive, and reversible stress/strain sensors with directional response and fast discoloration upon unloading.

The polymer used in the present work, $\mathrm{P}\left(\mathrm{SP}-\right.$ alt $\left._{-} \mathrm{C}_{10}\right)$, is potentially highly mechanoactive, since its main chain contains a spiropyran molecule in each repeat unit (Figure 1A). When compared to SP derivatives with an electron withdrawing nitro 

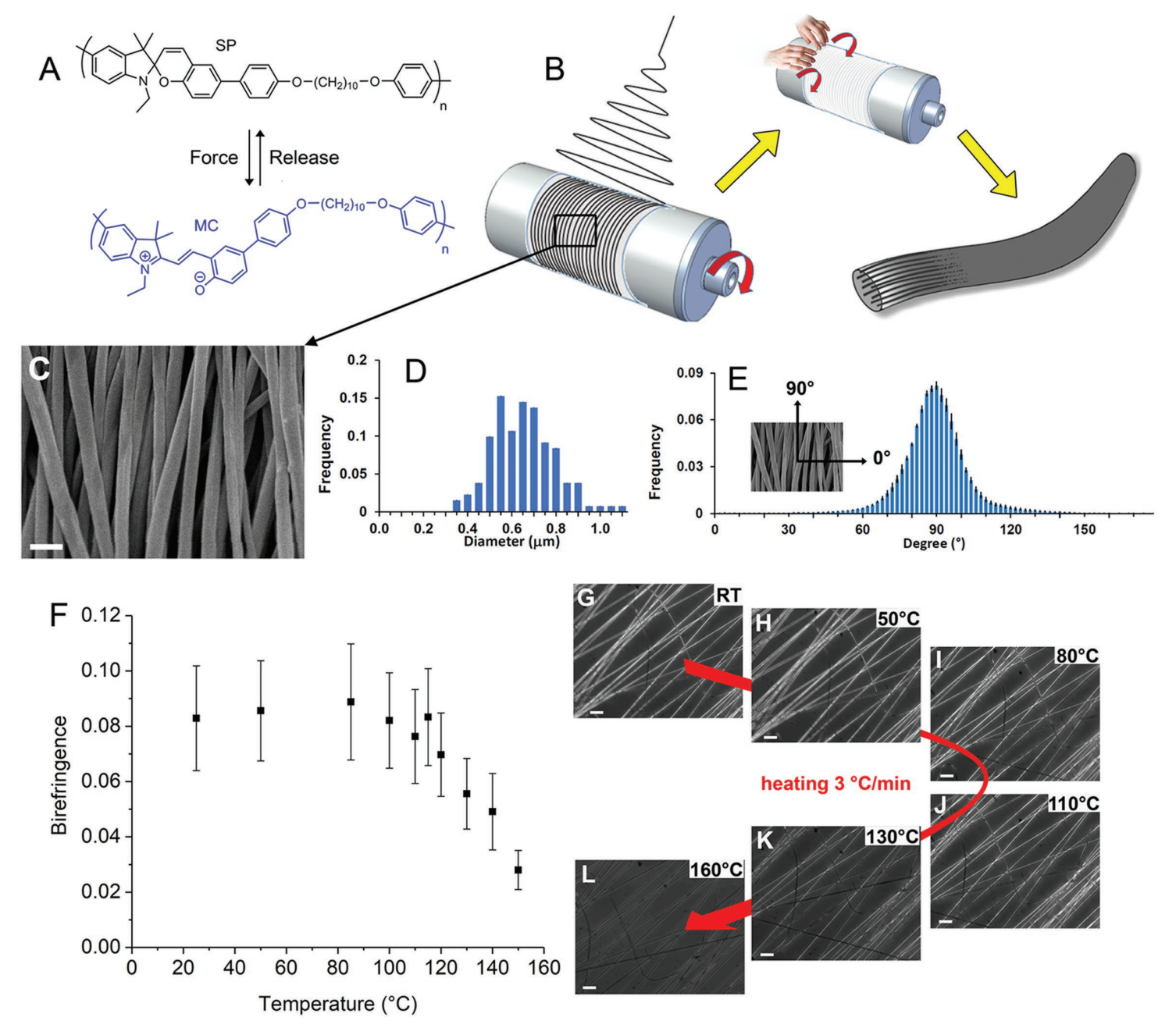

Figure 1. A) Chemical structure of $\mathrm{P}\left(\mathrm{SP}-a \mid t-\mathrm{C}_{10}\right)$ and its corresponding merocyanine form. B) Sketch describing $\mathrm{P}\left(\mathrm{SP}-\mathrm{alt}-\mathrm{C}_{10}\right)$ electrospun bundle fabrication: aligned fibers were collected on a high-speed rotating drum; the obtained mat was rolled-up to gain a bundle of fibers longitudinally aligned along bundle length. C) SEM image of the mat of aligned fibers used to produce the bundle (scale bar: $2 \mu \mathrm{m}$ ), D) The corresponding fiber diameter distribution and E) fiber orientation distribution. F) Birefringence of electrospun fibers thermally treated at different temperatures and $\mathrm{G}-\mathrm{L}$ ) gradual disappearance of birefringence in electrospun fibers heated at a controlled rate of $3{ }^{\circ} \mathrm{C} \mathrm{min}^{-1}$ (scale bar: $20 \mu \mathrm{m}$ ).

group, it allows overcoming two significant limitations that are typical for most reported SP copolymers. First, competing reactions initiated by other stimuli such as UV-light are less likely. ${ }^{[25,26]}$ Second, the low stability of the MC form causes immediate back reaction and makes the MC form only stable under maintained stress. ${ }^{[27]}$

The excellent solubility of $\mathrm{P}\left(\mathrm{SP}-a\right.$ alt- $\left.\mathrm{C}_{10}\right)$ allows electrospinning from dimethylformamide/tetrahydrofuran (DMF/THF) mixtures to produce fiber mats $(\approx 100 \mu \mathrm{m}$ thick) made of aligned, uniform, bead-free fibers (Figure 1C) with a mean diameter of $0.68 \pm 0.14 \mu \mathrm{m}$ (Figure 1D). The random deposition of fibers was prevented by collecting fibers on a drum rotating at $16 \mathrm{~m} \mathrm{~s}^{-1}$ that mechanically aligned the fibers along the drum circumference (Figure 1B,E). The aligned fiber mat was subsequently rolled-up ${ }^{[28]}$ to gain a fiber bundle with a thickness of $\approx 1 \mathrm{~mm}$ made of fibers well-aligned parallel to the bundle long axis (Figure 1B). The rationale behind using fiber bundles made from aligned fibers to investigate their mechanoresponse was: i) to test the polymer in the direction of fiber orientation, ii) to individually strain as much fibers as possible, and iii) to detect by naked eye any possible color change induced by the stretching. After electrospinning, the glassy state of the polymer was confirmed by wide-angle X-ray diffraction (WAXD) analysis showing an amorphous halo (Figure S2, Supporting Information) and by differential scanning calorimetry (DSC) ( $T_{\mathrm{g}}=120^{\circ} \mathrm{C}$, Figure S3, Supporting Information).

To evaluate possible chain orientation within fibers, the temperature-dependent birefringence of fibers was investigated. Indeed, the fibers showed birefringence with a maximum intensity at $45^{\circ}$ with respect to the direction of the polarizer and a minimum intensity at $0^{\circ}$ or $90^{\circ}$ (Figure S4, Supporting Information). This suggests a highly orientated glassy phase in the direction of the fiber axis. Electrospun fibers thermally treated for $10 \mathrm{~min}$ at different temperatures maintained their birefringence when kept at a temperature below $T_{\mathrm{g}}$. Conversely, higher heating temperatures provoked a significant decrease of birefringence that gradually reached a null value at $160{ }^{\circ} \mathrm{C}$ (Figure $1 \mathrm{~F}$ ). Consistent results were obtained by heating the fibers under a 
polarized optical microscope at a controlled heating rate from $\mathrm{RT}$ up to $160{ }^{\circ} \mathrm{C}$ (Figure $1 \mathrm{G}-\mathrm{L}$ ). The disappearance of birefringence, thus the loss of molecular orientation, occurs when crossing $T_{\mathrm{g}}$, namely when molecules acquire segmental mobility to relax and change their conformation from a stretched to a more coiled conformation. A further evidence of molecular orientation within $\mathrm{P}\left(\mathrm{SP}\right.$-alt- $\left.\mathrm{C}_{10}\right)$ fibers was provided by measuring the dimensional change of a fiber bundle with increasing temperature (Figure S5, Supporting Information). Fiber bundles exhibited shrinkage of more than $40 \%$ when crossing the $T_{\mathrm{g}}$, which is explained by chain relaxation during the glass-rubber transition. The presence of molecular orientation in electrospun fibers is the consequence of high shear rates $\left(100-1000 \mathrm{~s}^{-1}\right)$ and high draw ratios (up to 25000 ) that characterize the process, ${ }^{[24,29]}$ as widely demonstrated for semicrystalline polymers, ${ }^{[30-33]}$ but evidence of molecular orientation in fully amorphous polymers is rarely reported. ${ }^{[34,35]}$ In the latter case, vitrification of polymer chains in an out-of-equilibrium oriented state depends on chain relaxation time and solvent evaporation rate, and can only occur when the $T_{\mathrm{g}}$ of the polymer is above the temperature used for electrospinning. ${ }^{[29]}$ The herein used system and conditions for $\mathrm{P}\left(\mathrm{SP}\right.$-alt- $\left.\mathrm{C}_{10}\right)$ with $\mathrm{T}_{\mathrm{g}}=120{ }^{\circ} \mathrm{C}$ and electrospinning from a solvent mixture rich in low-boiling-point THF fulfill the requirements for chain orientation.

First qualitative investigations of the mechanochromic response of fiber bundles were done using stress-strain measurements. Figure 2A compares the stress-strain curves of a bundle and a solvent cast film and shows the corresponding images of samples acquired at different strain values. A first important result is the evident dramatic change of mechanical properties of the film compared to the fiber bundle. While the film shows brittle behavior and only reaches $10 \%$ strain before failure without exhibiting any color change, the same polymer displays a remarkably different behavior in electrospun fibers. The fiber bundle shows plastic deformation after $5 \%$ strain and can be deformed up to $70 \%$ before failure. Concomitantly, the bundle shows a strain-dependent intensity increase of the characteristic blue color of the MC form. By the naked eye, the color change appeared at $\approx 10 \%$ strain and was observed until failure. This transition from brittle to ductile behavior was previously observed for other polymers and is pivotal to reach sufficient deformation for mechanophore activation. Papkov et al. ascribed the toughness improvement in electrospun polyacrylonitrile fibers to low crystallinity that enhances the sliding mobility of long chains in the amorphous regions of the semicrystalline polymer. $^{[36]}$ The same hypothesis was proposed by Lu et al. to explain the surprisingly high elongation of semicrystalline polyoxymethylene electrospun fibers. ${ }^{[37]}$ However, the sharp rise in toughness was also reported for completely amorphous polymers such as polymethylmethacrylate ${ }^{[38]}$ and polystyrene. ${ }^{[39]}$ In these cases, the improvement in toughness was ascribed either to the presence of molecular orientation along the deformation, favoring interchain slippage and plastic deformation over tearing of covalent bonds, ${ }^{[40]}$ or to size-effect-driven defect
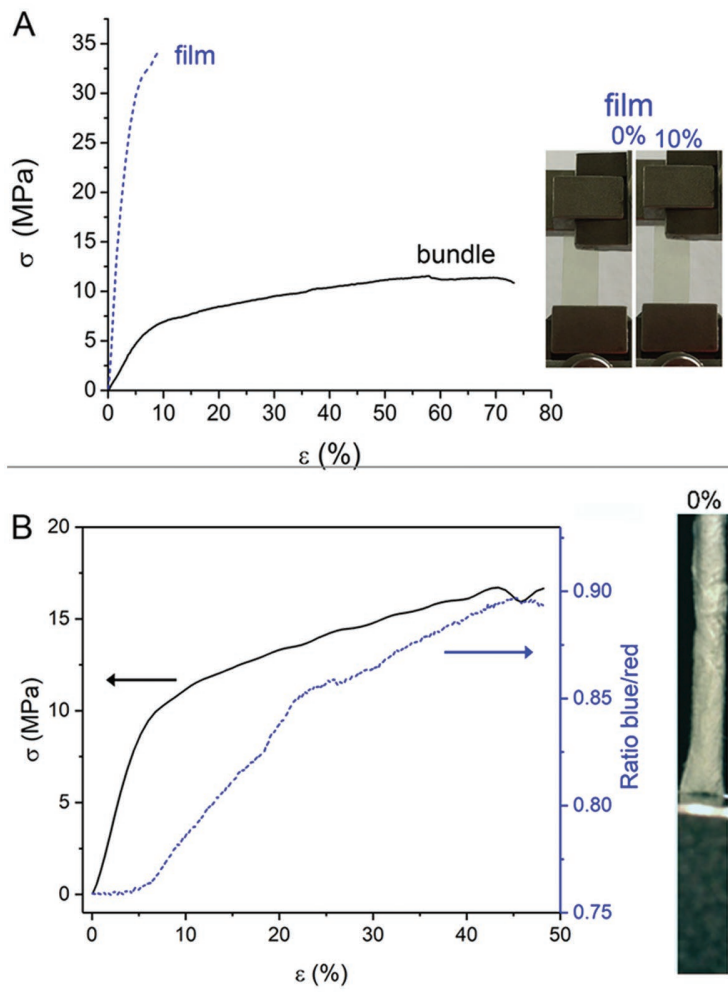

bundle
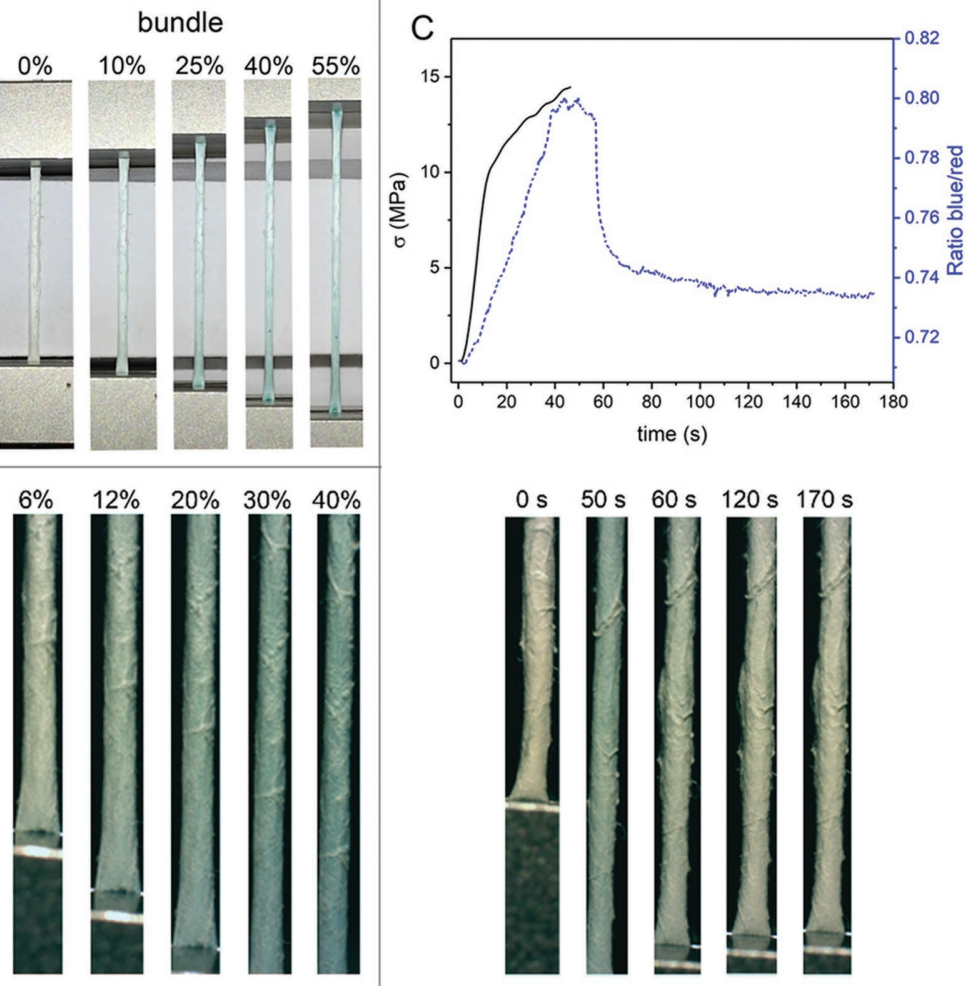

Figure 2. A) Stress-strain curves of film (blue dashed line) and electrospun bundle (black solid line) together with sample pictures at specific strain values. B) Stress-strain curve of electrospun bundle (black solid line), the observed intensity ratio of blue/red (blue dashed line) and sample pictures at specific strain values. C) Stress versus time curve of electrospun bundle (black solid line) during a stress-strain measure carried out at 5 mm min m $^{-1}$ up to $30 \%$ of strain, the intensity ratio of blue/red (blue dashed line) that was monitored up to 2 min after releasing the force, and sample pictures at specific times. 
removal in fibers with sub-micrometric diameters. ${ }^{[36,39]}$ For the presently investigated rather brittle polymer, the improvement of strain at break after electrospinning allows SP activation at low tensile strain, previously achieved for brittle polymers only when approaching $T_{\mathrm{g}}$ or after plasticization. ${ }^{[21,41]}$

A quantitative measure of color change was carried out by monitoring the ratio of blue/red colors of a defined area of the sample subjected to the stress-strain test. Figure 2B reports the blue to red ratio of the bundle as recorded by a CCD camera together with the corresponding stress-strain curve. During stretching, the blue color continuously increased until sample breaking. This phenomenon can be directly correlated to the increasing amount of mechanically isomerized merocyanine in the backbone of the copolymer chain. It can be noted, that at strain values below 5\% (in the elastic regime) the blue/red ratio remained constant and increased only after overcoming the yield point (around 5\% strain), with the occurrence of plastic deformation. Mechanoactivation at such a low value of strain is very uncommon for polymers based on the SP-MC transition. Typically, high deformations are required to align macromolecules and to transduce mechanical force to SP, being thus activated at strains higher than $100-200 \%,{ }^{[17,42]}$ often even higher than $300 \%[8,13,14,41]$ and sometimes demanding pre-strain. ${ }^{[16,18]}$ For $\mathrm{P}\left(\mathrm{SP}\right.$-alt- $\left.\mathrm{C}_{10}\right)$, the high SP content combined with the high degree of chain orientation enhance the mechanoresponse of the material remarkably and allow unprecedented results in terms of mechanoactivation to be achieved, especially if we consider that a brittle polymer was used.

An additional remarkable aspect in the mechanoresponse of the bundle is the sudden disappearance of the blue color upon force release. Figure 2C shows the results of another experiment where the bundle was deformed at a constant strain rate up to $30 \%$ strain. Then, the stress was released and the ring-closing reaction from $\mathrm{MC}$ to $\mathrm{SP}$ was followed by monitoring the blue to red ratio during time. After stress release the blue intensity drops in few seconds to a still positive value that, however, is no more distinguishable by the naked eye. This transient mechanochromic behavior is caused by the SP substitution pattern (Figure 1A) that lacks the MC stabilizing nitro group, ${ }^{[27,43]}$ and is ideally suited for dynamic real-time detection of stress and strain in, for example, elastomers. ${ }^{[17,25]}$

To further exploit the unique mechanochromic behavior of oriented fibers, nonwoven mats were used as fillers in an elastomeric PDMS matrix, given its importance for a variety of applications including, for instance, flexible electronics, microfluidic devices, and soft robotics. Using a simple procedure (Figure 3A,B), we prepared PDMS samples filled with either randomly oriented (Figure $3 \mathrm{C}$ ) or aligned $\mathrm{P}\left(\mathrm{SP}-\right.$ alt- $\mathrm{C}_{10}$ ) fibers (Figure 1C). An excellent matrix-fiber a dhesion ( Figure $3 \mathrm{D}$ ) enabled the effective transfer of the mechanical force from the matrix to the fibers. Figure 3E shows that PDMS filled with aligned fibers exhibited a remarkable increase of blue color at small strain values (around 5\% strain), in line with the mechanochromism displayed by the bundle (see Figure 2B). The same plot also reports the stress-strain (and the corresponding color change) of the same type of composite heated above $\mathrm{P}\left(\mathrm{SP}-\right.$ alt- $\left.\mathrm{C}_{10}\right) \mathrm{T}_{\mathrm{g}}$. After thermal treatment, a remarkable intensity decrease of the blue/red ratio was detected: at $40 \%$ of strain the color change was only one-third of the original composite. Moreover, albeit the heated composite withstood higher deformations before breaking (up to 100\%), it could not reach the same color change displayed by the original one. This less sensitive mechanochromism after thermal treatment can be ascribed to $\mathrm{P}\left(\mathrm{SP}-a l t-\mathrm{C}_{10}\right)$ chain relaxation occurring above $T_{\mathrm{g}}$ and confirms the hypothesis that chain orientation in electrospun fibers is responsible for the high sensitivity of mechanochromism displayed by this material.

A significantly different mechanochromic behavior was observed in the composite depending on fiber orientation. In particular, the use of randomly oriented fibers conferred mechanochromism to the composite in all stretching directions (Figure 3F,G, Figure 4A and Video S1, Supporting Information) whereas, with aligned fibers, the composite turned blue only when stretched in the direction of fiber alignment (Figure 3H,I, Figure 4A and Video S2, Supporting Information). Interestingly, quantitative assessment of color change as a function of strain (Figure 4A) yields a similar value of blue color for randomly oriented fibers and for aligned fibers stretched at $45^{\circ}$ with respect to the fiber axis (with a threshold strain to detect the increment of color of $10 \%$ ), which is also approximately the mean value between aligned fibers stretched perpendicular (almost zero color variation) or parallel to fibers axis (largest color variation with a threshold strain of $5 \%$ ). This indicates that the emergence of blue color is proportional to the alignment of the stress with respect to the fibers, and that such trend is approximately linear. Other authors have already proposed ad hoc synthetized responsive PDMS obtained by covalently bonding mechanophore units in the polymer network. ${ }^{[15,18,44]}$ However, the simple incorporation of highly mechanoresponsive fibers to produce mechanochromic PDMS-based composite brings a unique benefit: we can control the degree of fiber orientation, thus produce, for the first time to the best of our knowledge, materials with anisotropic mechanoresponsiveness. Remarkably, this impressive and clearly visible color change was achieved with only a 3 and $4.5 \mathrm{wt} \%$ of fiber content for random and aligned fibers, respectively.

Another remarkable property of the here presented PDMS-fiber composites is the reversible color change. As already discussed for polymer bundles, also in composites the SP-MC isomerization is reversible, and the blue color observed upon stretching the composite disappears when the stress is released and re-applied, even though some hysteresis is seen (Figure 4B). When the composite is stretched until forming a crack, a sudden decrease of the blue color is observed, which further decreases with crack propagation. Blue color disappears to the initial value when the material is completely broken (Figure 4B). The kinetics of coloration and decoloration is fast and allows for monitoring crack propagation in the material in real-time and in real-space, thus probing stress peaks and their release with high precision (Video S4 in true color and Video S5 in false color, Supporting Information).

In summary, we have demonstrated highly sensitive and directional stress/strain sensors from mechanochromic fiber/PDMS composites. What distinguishes the mechanochromic fibers from their bulk behavior is their higher sensitivity in terms of the required strain for color evolution enabled by chain orientation, which in turn is caused by the electrospinning process. Moreover, fiber bundles exhibit increased toughness, hence larger deformations are possible also for originally brittle polymers. These aspects render the concept of mechanochromic fiber composites 

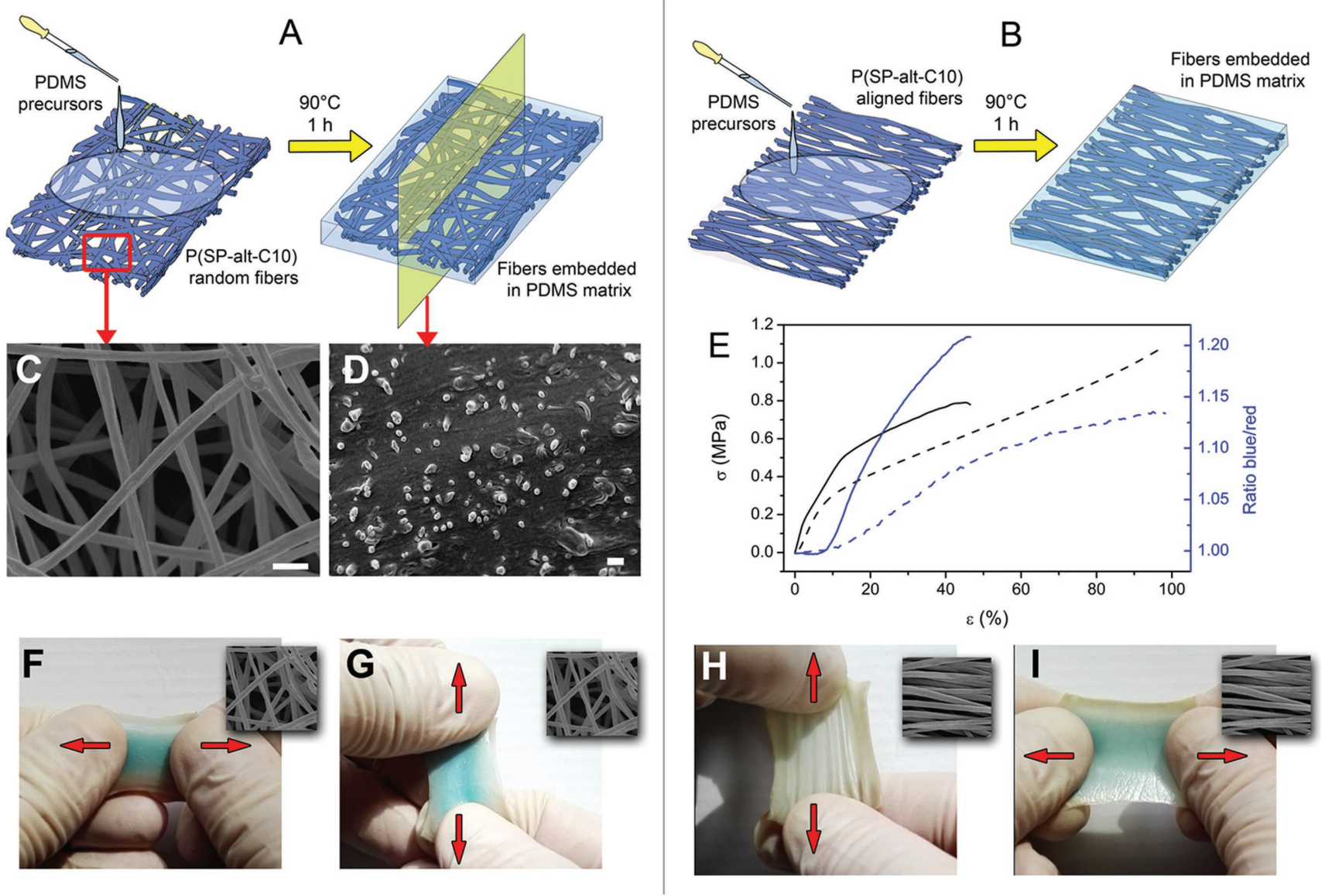

Figure 3. A,B) Sketches describing the fabrication of PDMS-fiber composites with a nonwoven mat of random and aligned fibers, respectively: the liquid mixture of PDMS precursors was gently poured on the nonwoven mats of $\mathrm{P}\left(\mathrm{SP}\right.$-alt- $\left.\mathrm{C}_{10}\right)$ and cured at $90^{\circ} \mathrm{C}$ for $1 \mathrm{~h}$. C) SEM image of $\mathrm{P}(\mathrm{SP}$-alt$\mathrm{C}_{10}$ ) random fibers used to produce the composite and D) SEM image of the composite section showing fibers embedded in the PDMS elastomeric matrix (scale bars: $2 \mu \mathrm{m}$ ). E) Stress-strain curves (black lines) of a composite filled with aligned fibers before (solid line) and after a thermal treatment at $150^{\circ} \mathrm{C}$ (dashed line) together with the corresponding observed intensity ratio of blue/red (blue lines). F,G) Pictures of the random fiber-composite manually stretched in two perpendicular directions (see Video S1, Supporting Information). H,I) Pictures of the aligned fiber-composite manually stretched perpendicularly to fiber direction $(\mathrm{H})$ and parallel to fiber direction (I) (see Video S2, Supporting Information). Red arrows indicate the direction of the applied force.
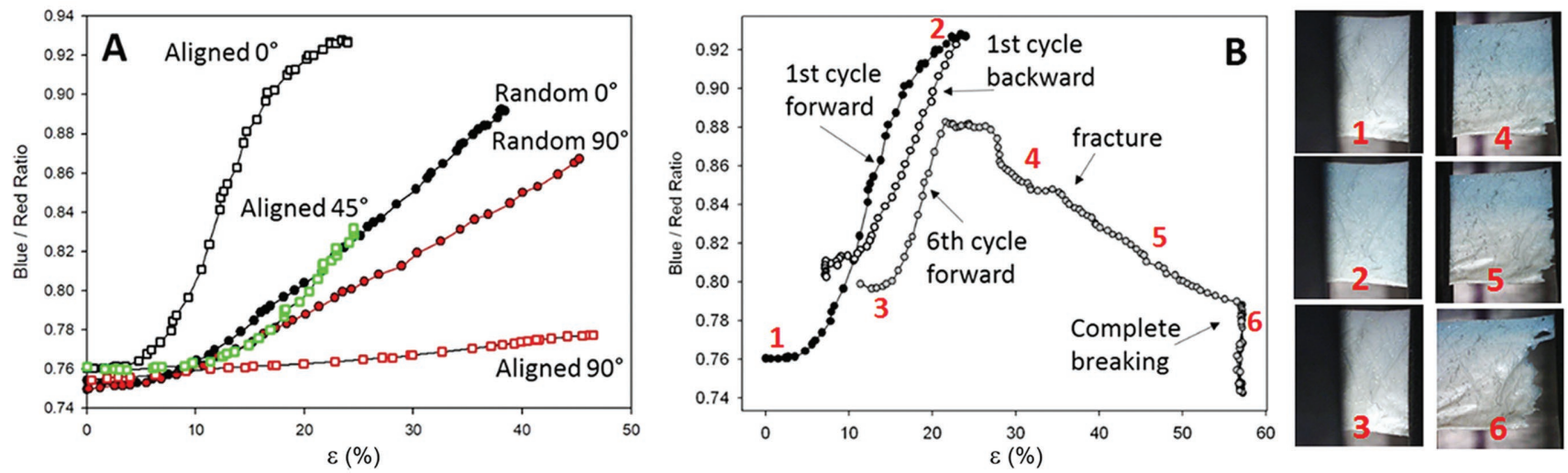

Figure 4. A) Color evolution of PDMS-fiber composites (expressed as the ratio between blue and red channels of an RGB CCD camera) as a function of the elongation in different directions: PDMS-fiber composites with randomly oriented fibers elongated in two perpendicular directions (black and red circles respectively) and with aligned fibers elongated along fiber axis $\left(0^{\circ}\right)$, at $45^{\circ}$ and perpendicular with fiber axis $\left(90^{\circ}\right)$ (black, green and red empty squares, respectively). B) Real-time evolution of the blue color (expressed as blue/red ratio) in a PDMS-fiber composite with aligned fibers during first elongation cycle (black and white circles for elongation and release, respectively) and during sixth elongation cycle which was continued until fracturing the composite and finally breaking it and corresponding sample pictures (1-6, see also Video S3, Supporting Information). 
unique and highly useful for other mechanochromic systems that, if properly processed by electrospinning, could show similarly beneficial behavior.

Undoubtedly, electrospun mechanochromic materials are highly attractive for a variety of applications in which fibers can be used as self-standing materials such as textiles or filtration membranes. Applications in the area of mechanobiology where electrospun, mechanoresponsive scaffolds can be used to investigate mechanotransduction phenomena are also possible. ${ }^{[3]}$ Of outmost interest is also their usage as fillers in composites where the possibility to control the directionality and the anisotropy of the strain-detection is expected to open further possibilities in the field of stress-sensing materials.

\section{Supporting Information}

Supporting Information is available from the Wiley Online Library or from the author.

\section{Acknowledgements}

The authors thank L. Xu for help with upscaling P(SP-alt- $\left.\mathrm{C}_{10}\right)$. M.S. acknowledges the DFG for funding (project SO 1213/7-1). The Italian Ministry of University and Research (MIUR) is acknowledged. Dario Cavallo is acknowledged for his assistance in birefringence measurements.

\section{Conflict of Interest}

The authors declare no conflict of interest.

\section{Keywords}

electrospinning, mechanochromic materials, spiropyran copolymers, stress-sensing

[1] F. Ciardelli, G. Ruggeri, A. Pucci, Chem. Soc. Rev. 2013, 42, 857.

[2] Y. Sagara, S. Yamane, M. Mitani, C. Weder, T. Kato, Adv. Mater. 2015, 28, 1073.

[3] Y. Jiang, Mater. Sci. Eng., C 2014, 45, 682.

[4] E. Ducrot, Y. Chen, M. Bulters, R. P. Sijbesma, C. Creton, Science 2014, 344, 186.

[5] S. Akbulatov, R. Boulatov, ChemPhysChem 2017, 18, 1422.

[6] S. Shree, M. Schulz-Senft, N. H. Alsleben, Y. K. Mishra, A. Staubitz, R. Adelung, ACS Appl. Mater. Interfaces 2017, 9, 38000.

[7] M. J. Robb, T. A. Kim, A. J. Halmes, S. R. White, N. R. Sottos, J. S. Moore, J. Am. Chem. Soc. 2016, 138, 12328.

[8] H. Zhang, Y. Chen, Y. Lin, X. Fang, Y. Xu, Y. Ruan, W. Weng, Macromolecules 2014, 47, 6783.

[9] R. Klajn, Chem. Soc. Rev. 2014, 43, 148.

[10] K. M. Wiggins, J. N. Brantley, C. W. Bielawski, Chem. Soc. Rev. 2013, 42, 7130.

[11] M. Li, Q. Zhang, Y. N. Zhou, S. Zhu, Prog. Polym. Sci. 2018, 79, 26.
[12] B. A. Beiermann, S. L. B. Kramer, J. S. Moore, S. R. White, N. R. Sottos, ACS Macro Lett. 2012, 1, 163.

[13] B. A. Beiermann, S. L. B. Kramer, P. A. May, J. S. Moore, S. R. White, N. R. Sottos, Adv. Funct. Mater. 2014, 24, 1529.

[14] X. Fang, H. Zhang, Y. Chen, Y. Lin, Y. Xu, W. Weng, Macromolecules 2013, 46, 6566.

[15] G. R. Gossweiler, G. B. Hewage, G. Soriano, Q. Wang, G. W. Welshofer, X. Zhao, S. L. Craig, ACS Macro Lett. 2014, 3, 216.

[16] C. K. Lee, D. A. Davis, S. R. White, J. S. Moore, N. R. Sottos, P. V. Braun, J. Am. Chem. Soc. 2010, 132, 16107.

[17] M. Li, W. Liu, S. Zhu, Polymer 2017, 112, 219.

[18] G. O'Bryan, B. M. Wong, J. R. McElhanon, ACS Appl. Mater. Interfaces 2010, 2, 1594.

[19] M. E. Grady, B. A. Beiermann, J. S. Moore, N. R. Sottos, ACS Appl. Mater. Interfaces 2014, 6, 5350.

[20] J. R. Hemmer, P. D. Smith, M. Horn, S. Alnemrat, B. P. Mason, J. R. Alaniz, S. Osswald, J. P. Hooper, J. Polym. Sci. Part B: Polym. Phys. 2014, 52, 1347.

[21] B. A. Beiermann, D. A. Davis, S. L. B. Kramer, J. S. Moore, N. R. Sottos, S. R. White, J. Mater. Chem. 2011, 21, 8443.

[22] J. W. Kim, Y. Jung, G. W. Coates, M. N. Silberstein, Macromolecules 2015, 48, 1335.

[23] T. van de Laar, H. Schuurman, P. van der Scheer, J. Maarten van Doorn, J. van der Gucht, J. Sprakel, Chem 2018, 4, 269.

[24] D. H. Reneker, A. L. Yarin, E. Zussman, H. Xu, in Advances in Applied Mechanics, Vol. 41 (Eds: A. Hassan, G. vander Erik), Elsevier, Amsterdam, The Netherlands 2007, p. 43.

[25] M. Li, Q. Zhang, S. Zhu, Polymer 2016, 99, 521.

[26] G. I. Peterson, M. B. Larsen, M. A. Ganter, D. W. Storti, A. J. Boydston, ACS Appl. Mater. Interfaces 2015, 7, 577.

[27] F. Kempe, O. Brugner, H. Buchheit, S. N. Momm, F. Riehle, S. Hameury, M. Walter, M. Sommer, Angew. Chem., Int. Ed. 2017, 57, 997.

[28] A. Sensini, C. Gualandi, L. Cristofolini, G. Tozzi, M. Dicarlo, G. Teti, M. Mattioli-Belmonte, M. L. Focarete, Biofabrication 2017, 9, 015025.

[29] M. Richard-Lacroix, C. Pellerin, Macromolecules 2013, 46, 9473.

[30] M. Gazzano, C. Gualandi, A. Zucchelli, T. Sui, A. M. Korsunsky, C. Reinhard, M. L. Focarete, Polymer 2015, 63, 154.

[31] M. V. Kakade, S. Givens, K. Gardner, K. H. Lee, D. B. Chase, J. F. Rabolt, J. Am. Chem. Soc. 2007, 129, 2777.

[32] J. Liu, D. Y. Lin, B. Wei, D. C. Martin, Polymer 2017, 118, 143.

[33] X. Ma, J. Liu, C. Ni, D. C. Martin, D. B. Chase, J. F. Rabolt, ACS Macro Lett. 2012, 1, 428.

[34] J. H. Wendorff, S. Agarwaj, A. Greiner, in Electrospinning: Materials, Processing, and Applications, Wiley-VCH, Weinheim, Germany 2012, p. 97.

[35] Y. Xu, Y. Gao, X. Wang, J. Jiang, J. Hou, Q. Li, Macromol. Mater. Eng. 2017, 302, 1700054/a.

[36] D. Papkov, Y. Zou, M. N. Andalib, A. Goponenko, S. Z. D. Cheng, Y. A. Dzenis, ACS Nano 2013, 7, 3324.

[37] J. W. Lu, Z. P. Zhang, X. Z. Ren, Y. Z. Chen, J. Yu, Z. X. Guo, Macromolecules 2008, 41, 3762

[38] X. Sui, H. D. Wagner, Nano Lett. 2009, 9, 1423.

[39] F. Zhang, A. H. Barber, Macromol. Mater. Eng. 2017, 302, $1700084 / a$

[40] I. Greenfeld, X. Sui, H. D. Wagner, Macromolecules 2016, 49, 6518.

[41] D. A. Davis, A. Hamilton, J. Yang, L. D. Cremar, D. Van Gough, S. L. Potisek, M. T. Ong, P. V. Braun, T. J. Martinez, S. R. White, J. S. Moore, N. R. Sottos, Nature 2009, 459, 68.

[42] S. Jiang, L. Zhang, T. Xie, Y. Lin, H. Zhang, Y. Xu, W. Weng, L. Dai, ACS Macro Lett. 2013, 2, 705.

[43] O. Brugner, T. Reichenbach, M. Sommer, M. Walter, J. Phys. Chem. A 2017, 121, 2683.

[44] N. A. A. Rossi, E. J. Duplock, J. Meegan, D. R. T. Roberts, J. J. Murphy, M. Patel, S. J. Holder, J. Mater. Chem. 2009, $19,7674$. 